Citation: R. Stella (2021) Effetti dell'astinenza dai media in un gruppo di studenti universitari. Media Education 12(2): 25-34. doi: 10.36253/ me-11577

Received: August, 2021

Accepted: October, 2021

Published: December, 2021

Copyright: @ 2021 R. Stella. This is an open access, peer-reviewed article published by Firenze University Press (http://www.fupress.com/me) and distributed under the terms of the Creative Commons Attribution License, which permits unrestricted use, distribution, and reproduction in any medium, provided the original author and source are credited.

Data Availability Statement: All relevant data are within the paper and its Supporting Information files.

Competing Interests: The Author(s) declare(s) no conflict of interest.

\section{Effetti dell'astinenza dai media in un gruppo di studenti universitari}

\author{
Effects of media abstinence on a group of college students
}

\author{
Renato Stella \\ Dipartimento FISPPA, Università di Padova \\ renato.stella@unipd.it
}

\begin{abstract}
The pervasiveness of new media has raised some questions as to what would happen if we were to deprive ourselves of different media forms for a shorter or longer time. The debate on this issue has become intense in recent years, especially in relation to the hypothesis that the relationship with the web and internet can configure a greater and more insidious dependence on media. To verify how, and if, this happens, I asked 121 university students to refrain from the consumption of media on smartphones, tablets, radios and televisions for a period ranging from three days to a week. The results, collected in diaries compiled during the test, testified to different levels of attachment to media, the interruption of which produced notable consequences in the daily organisation of time and social relations.
\end{abstract}

Keywords: old and new media, addiction, youth.

Riassunto. La pervasività dei nuovi media sollecita qualche interrogativo intorno a cosa accadrebbe se dovessimo privarcene per un tempo più o meno lungo. Il dibattito su una tale questione si è fatto intenso negli ultimi anni, in rapporto soprattutto all'ipotesi che la relazione con il web e internet possa configurare una dipendenza maggiore e più insidiosa rispetto a quella che conoscevamo con i vecchi media. Allo scopo di verificare come, e se, ciò accada, ho chiesto a 74 studentesse e 47 studenti universitari di astenersi dal consumo di smartphone, tablet, radio e televisioni per un tempo che va da tre giorni a una settimana. I risultati, raccolti nei diari compilati durante la prova, testimoniano livelli diversi di attaccamento ai media, la cui interruzione produce conseguenze notevoli nellorganizzazione quotidiana del tempo e delle relazioni sociali.

Paroile chiave: vecchi e nuovi media, dipendenza, giovani.

\section{SCIOPERI E DEPRIVAZIONE}

Studi sull'astinenza dai media, seppure rari nell'ambito delle ricerche che riguardano le comunicazioni di massa, tendono a riemergere ciclicamente. I primi esperimenti in questa direzione sono stati condotti a partire da even- 
ti fortuiti, che i ricercatori utilizzavano come contesti 'naturali' in cui valutare gli effetti di un'assenza prolungata di fonti di informazione. È così che Bernard Berelson (1949), in occasione di uno sciopero dei fattorini che distribuivano i giornali a New York nel 1945, pose le basi per quella che sarebbe poi divenuta la Teoria degli Usi e Gratificazioni (Blumler \& Katz, 1974). Su questa stessa traccia, ricerche successive approfittano di altre situazioni accidentali di sospensione delle reti comunicative per condurre delle osservazioni su pubblici di lettori e spettatori (De Bock, 1980; Cohen, 1981; Windahl, Höjerback, \& Hedinsson, 1986; Elliott \& Rosenberg, 1987).

Si scopre così quali sono i bisogni a cui i media rispondono e quali strategie i lettori di giornali, gli ascoltatori della radio e gli spettatori televisivi mettono in campo per sopperire alla loro mancanza. Fin da questi approcci si fa strada, nella prospettiva degli Usi e Gratificazioni, una prima idea di dependency e di affinity che lega i pubblici ai rispettivi media. Soprattutto la tv si presta a una tale interpretazione. L'astinenza forzata rende visibile la relazione intima e rituale col pubblico, non più osservata solo sotto forma di quantità di ore di consumo. Le reazioni di disagio, a seguito di una interruzione, la rendono invece più agilmente descrivibile negli effetti psicologici ed emozionali.

La questione diventa ancora più interessante quando si passa a considerare i media digitali. Web 2.0, social network, app e piattaforme di condivisione di testi e video, assumono un ruolo in buona parte diverso dai precedenti media in ragione del vincolo affettivo (Silva, 2012; Vincent, 2006; Paassonen, 2021; Mascheroni \& Vincent, 2016) o di dipendenza secondo altri (ad es. Lepp, Barkley, \& Karpinski, 2014; Clayton, Leshner, \& Almond, 2015) che apparecchiature, come il computer o lo smarphone, stabiliscono con i propri utenti in modi più tenaci e riconoscibili rispetto ai vecchi media. Intorno a questa biforcazione interpretativa si è sviluppato un dibattito, che in realtà dibatte poco, dal momento che $\mathrm{i}$ diversi approcci puntano a mantenersi su strade separate e poco comunicanti.

Ad esempio, le posizioni di stampo neurobiologico o psicologico cognitivo, maggioritarie per numero di articoli e ricerche, tendono a dare interpretazioni spesso drammatizzate. Il presupposto è che i nuovi media producano una mancanza (Scarcelli 2019), sia rispetto a un 'prima' che si giudica perduto (ad esempio la densità delle relazioni sociali in presenza), sia rispetto a un dopo che occorre costruire libero da deformazioni indotte da un eccesso d'uso. A questo infatti si associano ansia, depressione, bassa autostima e per l'appunto dipendenza in senso tecnico (vedi tra gli altri: Baker \& Perez Algorta, 2016; Chou, Condron, \& Belland, 2005). Tuttavia è evidente che un comportamento collettivo quasi universale non possa rientrare in una interpretazione generalizzante di questo tipo, come sottolineano, da punti di vista diversi, molti studiosi (boyd 2014; Ahn \& Jung 2016) Ci affideremo allora a un'idea di attaccamento ai nuovi media che non configura una forma di dipendenza in senso tecnico ma che, come vedremo, può comunque produrre conseguenze soggettive rilevanti, quali stress, ansia, senso di isolamento, anche in situazioni quotidiane del tutto ordinarie.

L'unica dimensione 'naturale' a cui si può fare riferimento per studiare stati di deprivazione dai media digitali, in assenza di episodi di blackout collettivo, sono i racconti delle occasioni in cui un qualsiasi accidente ne ha impedito l'utilizzo (rottura, smarrimento, impossibilità di connettersi). A questa prospettiva di ricerca si rifanno per esempio Hoffner, Lee \& Park, (2016), Paasonen (2021) e Lee et al. (2014). La raccolta a posteriori dei resoconti fa tesoro di riflessioni che rielaborano l'accaduto attraverso un processo di interpretazione personale, inducendo un più approfondito vaglio di emozioni ed effetti. Lo svantaggio è che la ricostruzione può essere lacunosa o parzialmente rifocalizzata su episodi locali e di minimo impatto.

Un po' più affollato invece il settore di indagine che, sin dagli studi sulla televisione (ad esempio: Knowles et al., 1989; Tan, 1977) provvede a creare delle condizioni simulate di privazione da uno o più media, su base volontaria e per un tempo definito, modello al quale fa riferimento anche la nostra indagine. Si giunge allora alla consapevolezza che i media tradizionali (stampa, tv, radio), allargano il raggio della loro influenza fino a diventare strumenti tanto indispensabili alla soddisfazione di bisogni individuali da creare dei problemi se vengono a mancare per più di qualche giorno (vedi le classiche ricerche legate al modello della dipendenza televisiva di Ball-Rokeach \& DeFleur 1976; Rubin \& Windahl, 1986).

Per venire agli studi più vicini a noi, Tromholt, (2016); Stieger \& Lewetz, (2018); Hall et al. (2021) hanno avviato esperimenti di astinenza dai social; Cheever et al. (2014) dal cellulare, mentre il più interessante si è rivelato il lavoro di Moeller, Powers, \& Roberts, (2012) che prevedeva il coinvolgimento di studenti universitari impegnati a privarsi dell'uso di qualsiasi medium per $24 \mathrm{~h}$. I risultati mostrano due tendenze generali che possiamo considerare uniformi tra le varie ricerche, compresa la nostra. La prima riguarda la propensione, da parte dei soggetti coinvolti nell'esperimento, ad autorappresentarsi come dipendenti, addirittura a volte drogati, dai media, tanto da manifestare disagio e da esprimere il desiderio di limitarne l'uso. L'idea che i media digitali 
sviluppino forme di assuefazione percepita individualmente per tale, è molto diffusa, in parte perché costituisce un leitmotiv della narrazione mediale e del discorso di senso comune, soprattutto adulto (Scarcelli, 2019), dall'altra perché l'impostazione dell'esercitazione, come meglio diremo tra breve, presuppone la dipendenza come situazione di partenza.

La seconda tendenza generale concerne il sostanziale ritorno alla normalità dopo concluso l'esperimento, con poche, anche se rilevanti, modifiche alle routine d'uso di app e social. Il che dimostra un potente attaccamento ai media digitali e ai loro contenuti, legato in parte al medium in sé e alla sua insostituibile onnipotenza nel fornire informazioni, ma in parte maggiore ancorato alla rete di connessioni sociali che rende attive e attingibili come bagaglio dell'esperienza di vita.

È questo il quadro in cui vorremo inserirci cercando di valutare la questione su base empirica, entro una cornice interpretativa che ponga l'ambivalenza (Grossi, 2017) come criterio portante. Vale a dire, l'idea che non si possa ragionare nei termini di una perdita (di attenzione, di tempo, di relazioni) che soprattutto i social imporrebbero rispetto alla situazione precedente la loro comparsa. I nuovi media, infatti, sovente integrano e allargano l'ambito del possibile delle interazioni sociali in contesti di massima condivisione, stabiliscono delle «infrastrutture di intimità» per stare alle parole di Paasonen (2021), entro cui nascono e si sviluppano amicizie, affetti e incontri sessuali, cioè il sale della socialità, soprattutto tra i giovani. In tale prospettiva, i nuovi media sono tecnologie indispensabili, tanto quanto lo sono i trasporti o i cavi elettrici. Di qui la necessità di porsi analiticamente in una prospettiva non lineare e ambivalente, che restituisca la complessità e la contraddittorietà di comportamenti collettivi non omogenei.

L'ambivalenza tocca allora il fatto, ovvio per le scienze sociali, che vi sono perdite e guadagni che si articolano in rapporto alle negoziazioni che sovraintendono alle fitte reti di rapporti tra gruppi e individui costantemente connessi tra loro. Esiste, alla fine, un diffuso attaccamento ai nuovi media, che si può anche definire 'dipendenza', ma che in nessun modo richiama sintomi vicini alle forme di assuefazione chimica o comportamentale studiati in psichiatria.

\section{LA RICERCA}

\subsection{Metodo}

Negli anni accademici 2017-2018 e 2018-2019 ho proposto agli studenti e alle studentesse del corso di Comunicazione di Padova di astenersi, per una settima- na, dal loro uso abituale dei media. La 'sfida' riguardava tutte le forme di comunicazione disponibili, ma è stato lasciato loro un ampio spazio di decisione per quanto riguarda la scelta dei media tradizionali e digitali di cui privarsi e dei tempi di durata dell'esercitazione. Il che ha rappresentato, come vedremo, uno degli elementi di novità rispetto agli studi precedenti.

Ho poi chiesto loro di compilare un diario, giorno per giorno, descrivendo le reazioni, i pensieri, le conseguenze e le difficoltà che l'esperimento comportava. La prova si concludeva con la scrittura di un paper in cui presentare e discutere i dati salienti emersi. L'esercitazione non era obbligatoria, ma posta in alternativa a un'altra, che consisteva nella mappatura mediale della propria famiglia (quante $\mathrm{tv}$, radio, riviste; quale distribuzione giornaliera degli usi tra i familiari ecc.). Un compito, questo, decisamente e volutamente meno impegnativo. Entrambe erano compensate con un massimo di 2 voti per l'esame finale.

I diari raccolti alla fine sono stati 129 . Ho escluso sette studenti lavoratori che superavano di gran lunga il range di età 18-22, maggioritario tra le matricole; un diario è stato scartato perché mancante di alcuni dati essenziali. In conclusione, ne ho ritenuti validi 121 , redatti da 47 ragazzi e 74 ragazze. La differenza di adesioni tra studenti e studentesse rispecchia la distribuzione diseguale degli iscritti al corso di laurea di Comunicazione a Padova, tuttavia lo sbilanciamento di genere è leggermente maggiore rispetto alle matricole nei due anni considerati. ${ }^{1}$ Se ne deduce che le più interessate ad affrontare la 'sfida' siano state le ragazze, come, forse, anche le più motivate a impegnarsi in una prova complessa rispetto a quanto era offerto in alternativa.-

\subsection{La sfida}

Porre la questioni in termini di 'sfida' («sareste capaci di...»), presuppone implicitamente che non sia facile separarsi dall'uso abituale dei media e suggerisce una forma di attaccamento che gli studenti spesso denunciano già prima di dare inizio alla prova. La consapevolezza di questa relazione intima col medium per molti non costituisce, allora, uno degli esiti dell'esperimento, ma una premessa condivisa, probabilmente anche uno sti-

\footnotetext{
${ }^{1}$ I diari sono stati stilati per il $38,8 \%$ (47) da ragazzi e per il $61,2 \%$ (74) da ragazze; mentre gli iscritti e le iscritte a Comunicazione al primo anno, nei due anni considerati, sono rispettivamente il $43,3 \%$ (141) e il $56,7 \%$ (185) (il mio corso è al primo semestre del primo anno, quindi i dati relativi alle matricole definiscono l'universo da cui è tratto il campione). In totale il gruppo dei 121 studenti e studentesse rappresenta il $37,1 \%$ di tutte le matricole iscritte al corso nei due anni, segno che la 'sfida', oltre a stuzzicare alcuni, ha spaventato molti...
} 
molo ("vediamo se ne sono capace di...»), che viene confermata più volte nei diari. Sapendo di essere, seppure in una maniera ancora confusa, 'dipendenti', ci si riconosce più motivati ad accettare la sfida, allo scopo di valutare fino a che punto lo si sia poi per davvero.

Come abbiamo anticipato, ciascuno studente o studentessa poteva configurare la sua partecipazione secondo le proprie aspettative e abilità. Programmare le cose in un modo piuttosto che in un altro, combinando la scelta dei media da cui astenersi con quella dei tempi, rivela già delle attitudini e delle previsioni di ordine sia tecnico, sia emotivo. In termini tecnici si è trattato di valutare ciò che dei diversi media 'davvero serve', escludendo alcune delle loro funzioni dal progetto dell'astinenza. Ad esempio, impegnarsi a non usare il cellulare, ma mantenere la possibilità di ricevere o fare chiamate con i genitori essendo fuori sede; comprendere tra $\mathrm{i}$ media esclusi il computer, ma garantirsi di poterlo usare per scrivere i paper e per consultare i siti di ateneo; in particolari circostanze, poi, assicurarsi la eventualità di comunicare, qualunque sia il medium, per seguire attività sportive di allenamento o piani sanitari. Il tutto testimonia una capacità organizzativa e previsionale meditata, con la quale si sono misurate le proprie forze e le proprie esigenze alla vigilia di un compito che si reputava impegnativo.

In termini emotivi, la diversa distribuzione dei media da cui astenersi e dei tempi, segnala le preoccupazioni intorno alla propria resistenza e alla probabilità di raggiungere l'obiettivo che ci si prefigge. Modulare la proposta in forma prudente, oppure generosamente azzardata, denota, come per le scelte tecniche, una certa capacità di razionalizzare in alcuni e un gusto giocoso e impulsivo per la sfida in altri. ${ }^{2}$

Si apre così un panorama di scelte, di per sé significativo, intorno all'idea che ciascuno ha del proprio rapporto coi media, e ciò ancor prima di cominciare in concreto l'esercizio. In altri termini, se e quando il legame con un medium definisce anche una forma percepita di dipendenza, questa è sottintesa nell'azione di scelta dei tempi e del mezzo da cui astenersi. La dipendenza non è, allora, solo un'idea di senso comune interiorizzata, ma, nell'esperimento proposto, si trasforma per molti, anche se non per tutti, in una pratica tecnica di selezione. Il sentore soggettivo dell'attaccamento a ciascun medium è parte dell'esperienza di consumo pregressa e, in tale veste, condiziona l'adesione alla prova articolandola secondo le capacità di giudizio e le aspettative personali. Una prima conseguenza rilevante è la riduzione del tasso

\footnotetext{
${ }^{2}$ Alla fine 81 tra studenti e studentesse (il 66,9\%) sceglie una settimana o più di astinenza, mentre 65 (il 53,7\%) si privano di tutti i media, alcuni con qualche eccezione tecnica.
}

di insuccesso. Potendo porre la questione a misura delle proprie forze è stato anche più facile mantenere l'impegno preso.

\section{GIOVANI ASTINENTI}

Dalla somma davvero consistente di dati e informazioni che emergono dai diari, prenderò ora in esame le osservazioni e i commenti più significativi. Per comodità di esposizione e limiti di spazio procederò per punti. , È interessante notare che questi in parte ricalcano quelli discussi da Dardenne nel 1994, relativi a un esperimento di deprivazione condotto prima della nascita del web. La coincidenza parziale dei resoconti, a distanza di 30 anni e con risorse tecnologiche diverse, mostra l'esistenza di un nucleo di relazioni tra media e pubblici giovanili che non è cambiato nel tempo e rispetto al quale i media digitali sembrano aggiungersi, dilatandone gli effetti ${ }^{3}$.

\subsection{Il medium}

Lo stato di 'dipendenza' è percepito soggettivamente, e raccontato nei diari, attraverso i gesti automatici che, soprattutto nei primi giorni, si compiono nei confronti del cellulare, della radio o del telecomando della tv e che vengono immediatamente corretti non appena ci si ricorda che si sta svolgendo un esercizio di astinenza che lo vieta. «Ho potuto osservare che la dipendenza dai media è visibile soprattutto dai gesti meccanici, dai gesti abituali che una persona è abituata a fare» (Te13-18). ${ }^{4}$

Allo scopo di essere al sicuro rispetto alle tentazioni e alle routine che producono azioni istintive, vengono introdotte strategie diverse: disconnettersi da app e social in modo da non ricevere notifiche e messaggi; tenere il cellulare lontano da sé, in luoghi inaccessibili, oppure a casa quando si esce; addirittura esportare «la scheda sim del mio Samsung del 2017 ad un Nokia di 10 anni fa utilizzando solo le funzioni base: chiamate e sms» (Sa19-19). La percezione del legame fortissimo che rende difficoltoso trovare un modo adeguato di privarsi dell'uso del cellulare è una costante valida per tutti, segno che l'idea di 'dipendenza', denunciata da molti

\footnotetext{
${ }^{3}$ Dardenne riportava che i media offrono compagnia, facilitano i rapporti sociali, spesso creano condizioni di dipendenza (addiction) percepita soggettivamente, si usano per eliminare il silenzio, la noia e persino il pensiero, tutte caratteristiche che ritroviamo commentate, insieme ad altre, nei diari.

${ }^{4}$ La sigla che identifica l'autore/autrice della citazione è così composta: "Te" individua uno studente, "Sa" una studentessa, seguono poi il numero progressivo attribuito al diario e l'età. La sigla nel testo si legge pertanto: il diario numero 13 scritto da uno studente di 18 anni.
} 
all'inizio della prova, deve essere contrastata in maniera decisa ed efficace. «Dunque mi sono presa in carico di provocarmi volontariamente una sorta di crisi di astinenza, proprio come quella di un tossicomane indotto a diminuire le sue dosi di droga» (Sa36-20).

Queste precauzioni però servono a poco rispetto alle mille funzioni che uno smartphone possiede e delle quali si avverte la mancanza via via che si procede con la prova «[il cellulare] era diventato la mia sveglia, la mia agenda, la mia rubrica, il mio personal trainer, il mio ricettario, il mio shopping (online), il mio mp4 e pure la mia tv!» (Sa48-19), tanto che la stessa studentessa, per compensare la mancanza, prende una decisione drastica «Domani devo comprare un orologio!». Occorre dunque ingegnarsi a sopperire a una parte almeno di quel che viene perduto, ricorrendo ad altri media (un vecchio $\mathrm{mp} 3$ o lo stereo di papà per la musica, il telefono fisso di famiglia per gli appuntamenti, il racconto degli amici per l'esito delle partite di calcio della squadra del cuore ecc.). L'elenco delle 'scoperte' a questo proposito, come vedremo, è piuttosto lungo e comprende anche strani gap generazionali:

I vecchietti del bar sono rimasti sorpresi quando gli ho spiegato del mio progetto di astinenza e mi hanno raccontato di come si stava 50 anni fa, quando non esistevano telefoni cellulari e la gente si incontrava sempre al solito posto, quando le ragazze le potevi vedere una volta alla settimana, la domenica pomeriggio in discoteca e passavano mesi prima di poterle chiedere il numero (di casa!) (Sa18-20)

\subsection{Conseguenze sociali}

Le conseguenze del periodo di astinenza riguardano sia lo stato di benessere personale, sia i rapporti con gli altri, che si complicano, non solo per le difficoltà dovute al non essere più connessi attraverso app e social, ma perché si vive in famiglia o con coinquilini, che occorre in qualche modo coinvolgere. Per prima cosa è necessario decidere se dare o no la notizia ad amici e parenti. «Ho consapevolmente deciso di non avvertire nessuno, per vedere, al mio ritorno, quante fossero le persone che avrebbero notato la mia assenza» (Sa18-20). Posizione, questa, condivisa da pochi altri, ma orientata a fare dell'esercitazione una sorta di sondaggio intorno alla consistenza della rete di relazioni che si mantengono attraverso il web e che spesso, si scoprirà, produce risultati deludenti.

Nella maggior parte dei casi si preferisce invece informare della novità: «Abbiamo messo come foto profilo di WhatsApp, una scritta avvisando che per qualche giorno non utilizzeremo Internet» (Sa97-19). Alla notizia di solito si ottengono commenti di incredulità o di inco- raggiamento da parte dei coetanei «[Gli amici] dopo aver spiegato loro della mia astinenza, mi guardano con gli occhi di chi osserva con compassione un malato grave e quasi mi fanno le condoglianze» (Te93-19); «Se lo fai davvero ti stimo! Io non riuscirei nemmeno per un giorno!» (Sa100-20). Ciò in linea con l'idea diffusa e implicita che i nuovi media creino dipendenza e che, come sostiene uno studente, «essendo io un ragazzo normale passo la maggior parte delle mie giornate online» (Te1222). In altri termini, se la dipendenza esiste, questa rientra nei parametri di un comportamento atteso che definisce i confini stessi della normalità.

Un secondo ambito di relazione che occorre gestire durante l'astinenza riguarda i conviventi, familiari o coinquilini. Qui si seguono due diverse strategie: si cerca di coinvolgerli in modo da rendere meno accidentato il proprio percorso (18 diari riguardano più persone che partecipano alla prova) $)^{5}$; oppure si tenta di imporre, a genitori e amici, i propri obblighi quando si è in compresenza. I genitori di solito non reagiscono molto bene e sopportano malvolentieri di non accendere la tv, fino a confinare il proprio figlio o la propria figlia in camera durante i pasti: «la mia famiglia non ne voleva sapere di spegnere la televisione (...) di conseguenza sono andata a mangiare in camera mia» (Sa44-19). Vi sono anche espliciti rifiuti di fornire la propria collaborazione: «Avrei apprezzato molto se il mio ragazzo, la mia famiglia, le mie amiche avessero partecipato, ma hanno preferito restarne fuori» (Sa100-20). Insieme a repentini cambi di rotta: «il mio fidanzato sta iniziando a pentirsi di questo esperimento e si è arrabbiato un po' con me perché gli ho proposto con entusiasmo questa esperienza (Sa97-19)».6

Le conseguenze maggiori, tuttavia, toccano le relazioni mediate dalle app e dai social che vengono interrotte, rendendo ardue anche le situazioni abituali più semplici. «Questi 'veri' amici però, sono letteralmente spariti durante questi sette giorni, nemmeno un messaggio, una chiamata, né uno squillo, come se da un giorno all'altro io non esistessi più» (Te8-19). Saltano appuntamenti, è difficile gestire i ritardi, senza connessione nessuno si preoccupa di ricorrere a media alternativi per parlare con te anche se è il tuo compleanno. Naturalmente, qui come altrove, molto dipende da come si è organizzata la prova sin dal primo giorno e, come dicevamo, i più avveduti (soprattutto le più avvedute) sono anche quelli che hanno meno problemi.

\footnotetext{
${ }^{5}$ Nello specifico, 12 diari coinvolgono altri componenti della famiglia (genitori, nonni, sorelle o fratelli); 4 diari amici o coinquilini, infine 1 diario il fidanzato e 1 diario la fidanzata.

${ }^{6}$ Naturalmente, tutte queste considerazioni sono prodotte a seguito di una interpretazione radicale dellastinenza dalla tv. Alcuni, infatti, in previsione di problemi simili, avevano programmato di non coinvolgere nessuno e di seguire la tv con la famiglia durante i pasti.
} 
Una importante notazione, relativa ai rapporti sociali, riguarda l'effetto di proiezione di sé sui comportamenti altrui, una volta che se ne sia stati privati. È la scoperta del mondo del web visto da fuori, che sorprende e incupisce.

Entro in aula (...) mi guardo intorno e mi accorgo che tutta la cinquantina di miei compagni di corso presenti aveva il cellulare in mano. Ma la cosa che mi stupisce non è tanto questa, ma il fatto che prima d'ora non me ne n'ero mai accorta, questo perché fino al giorno prima con il cellulare in mano dentro l'aula c'ero anch'io (Sa58-18) Oggi ho potuto sperimentare un fatto interessante. In ogni luogo che ho attraversato nell'arco della giornata, dal vagone del treno, alla stazione, dalla strada al bar, la maggior parte delle persone era completamente immersa nel proprio mondo mediatico. (Sa78-19)

La constatazione che quel che accade adesso agli altri, prima coinvolgeva anche me senza che me ne rendessi conto, rafforza l'idea della dipendenza. È l'unico modo per interpretare un comportamento collettivo di cui ci si accorge solo per il fatto di esserne esclusi:

Ho osservato molto le persone che mi circondano e ho potuto trarre una conclusione molto importante: la gran parte delle persone è dipendente, a mio avviso, a livelli quasi patologici, dallo smartphone, me compreso. (Te87-19).

Non nascondo che passare un'ora con una persona che per il settanta percento del tempo sta a guardare il telefono è molto stressante e comunque ho notato di quante cose ci si accorge guardando le persone e non lo schermo del cellulare (Te12-22).

Mantenere una distinzione tra il dentro e il fuori della rete, soprattutto per coloro che usano molto spesso il web, è metodologicamente poco appropriato, tuttavia il nostro esperimento gli restituisce una certa visibilità e permette a molti ragazzi e ragazze di constatarlo per via empirica. Queste considerazioni generali rientrano negli effetti non attesi dell'astinenza: si prende coscienza delle routine quando vengono a mancare e si capisce, per estensione, di far parte della stessa massa informe di persone «con la testa chinata a guardare il proprio dispositivo» (Te87-19). Ma non tutti vivono una tale scoperta nel medesimo modo. C'è chi, di fronte alla scena, prova nostalgia e vede aumentare la sensazione di isolamento «in treno mi sento particolarmente escluso: dai ragazzi della mia età ai signori di età più avanzata, la maggior parte di loro tiene in mano un cellulare» (Te4219). L'esito ambivalente della situazione gioca a livello della 'normalità' a cui accennava lo studente poco prima. I più si sorprendono di quanto sia normale un comportamento collettivo che, visto dal di fuori, fa impressione e sconcerta; alcuni invece vorrebbero riappropriarsi di quella stessa normalità che è stata dolorosamente interrotta e di cui sentono la mancanza.

Effetto della scoperta di come sopravvivere 'fuori dal web' è anche l'insieme di occasioni di contatto che si aprono grazie al tempo liberato. In autobus, in aula, nelle file d'attesa, si attacca bottone con perfetti sconosciuti, ricavandone sensazioni inedite e qualche motivo di orgoglio «In treno continuo a parlare con gente a me sconosciuta, sono diventato un chiacchierone e questa cosa non mi dispiace affatto» (Te87-19). L'alternativa all'«osservare la campagna veneta dal finestrino» (Te9319) per «ore che si rivelano interminabili» (Te42-19) è ascoltare chi ti sta intorno, addirittura fantasticare sulle loro vite e su ciò che raccontano, oppure prendere l'iniziativa e ritrovarsi capaci di una agency sociale che prima non ci si riconosceva.

Un amico mi ha fatto notare che parlo, intervengo e cerco di mantenere attiva la conversazione di più rispetto a prima, quando ci si perdeva sul telefono, devo dire che mi fa piacere perché per il mio carattere può essere un grande cambiamento! (Te107-22)

\subsection{Conseguenze personali}

In termini soggettivi, gli stati di ansia, stress, preoccupazione e noia sono denunciati dai più. Ma vi sono anche esiti che appaiono opposti. Il sonno, per esempio, l'attenzione per lo studio, la capacità di concentrazione variano da un soggetto all'altro. Per alcuni è più facile addormentarsi e ci si sveglia più riposati grazie al fatto di non usare lo smartphone a letto la sera. Addirittura, migliorano le condizioni degli occhi «Diminuisce il bruciore agli occhi causato dalla luce blu degli schermi (sindrome da visione del computer)»(Te13-18). Ad altri accade il contrario: «Sono curioso di sapere come riuscirò ad affrontare la fase di rilassamento a letto (...) che mi concilia il sonno, senza aver accesso a Instagram e a YouTube» (Te86-19). Gli effetti fisici sono di vario tipo, fino a toccare situazioni molto intime e personali: «Non avendo accesso al web la pulsione sessuale è scesa in maniera vertiginosa, la voglia di praticare autoerotismo è praticamente svanita» (Te43-21), «Andare al bagno per la 'seduta quotidiana' senza cellulare e senza poter leggere un giornale mi fa riflettere» (Te42-19) (notazioni di questo tipo appaiono più volte nei diari). Al di là del valore degli episodi, è notevole il livello di confidenza che il racconto esprime, insieme al candore con cui, qui come altrove, si 'sperimentano' comportamenti inediti, una volta che ci si sleghi dalla pervasività dei media digitali che hanno raggiunto, possiamo dire con buone 
ragioni, ormai davvero qualunque ambito della vita.

Dardenne ricorda che i suoi studenti lamentavano silenzio, noia e pensieri come conseguenze psicologiche da gestire in assenza dei media. Ritrovo considerazioni simili nei diari. La noia si accompagna soprattutto al tempo libero. Ogni situazione che comprenda un'attesa: il viaggio in treno o in autobus, guidare la macchina, la fila in biglietteria, senza poter ascoltare musica diventa una tortura. "Palestra senza musica e senza serie tv è stata infinita. 2 ore di treno senza musica + un'ora di macchina senza radio = sofferenza» $($ Sa48-19). Una lamentela che si ripete quasi con tutti e che riprende uno dei disagi più sentiti e variamente espressi. A questo si aggiungono, in generale, i tempi morti durante le normali attività della giornata.

La liberazione del tempo stimola tuttavia l'esplorazione di alternative, e qui si apre il vaso di Pandora della ricerca di riempitivi: si va dal giocare a carte con mamma o fidanzato, al rimette a posto l'armadio; dal riprendere in mano la chitarra o la batteria dopo anni o mesi di inattività, al disegnare paesaggi; dal comporre musica, al fare esercizio fisico; dal passeggiare per le strade della propria città, al guardare vecchie fotografie. Ma la parte del leone spetta alla lettura. Leggere un libro diventa l'equivalente di ogni altro svago perduto.

Ero scettico sul fatto che leggere un libro potesse divertir$\mathrm{mi}$. Sono rimasto invece stupito da come la lettura abbia intrattenuto la maggior parte delle mie serate durante la settimana, stimolando molto la mia immaginazione.

(Te122-19)

Accade anche l'imprevedibile: «visto che non ho internet sono andato a comprare un quotidiano per la prima volta in vita mia» (Te80-19).

I pensieri, lo scorrere di riflessioni su di sé, sono uno degli effetti dell'assenza riempitiva e distraente dei media. Soprattutto se si tratta di meditazioni inopportune: «Un effetto molto rilevante che ho notato per tutta la settimana è stato l'aumento dei pensieri (...) Prima se stavo pensando a qualcosa di negativo accendevo la tv oppure il computer e mi distraevo» (Sa99-20). Anche guardare fuori dal finestrino viaggiando in treno produce lo stesso effetto e seppure i pensieri non sono necessariamente sempre negativi, si tratta comunque di una situazione inaspettata e faticosa "ho sentito molto la mancanza della musica per fermare il flusso costante dei pensieri» (Te81-20).

Il vuoto e il silenzio sono altri aspetti della liberazione di tempo: «Non sono più abituato ad annoiarmi e mi dà un senso interiore di vuoto pazzesco, prima ero sempre occupato a fare qualcosa sul telefono, o un video o nuove notifiche sui social; ora dovrei utilizzare il tempo per me» (Te107-22). Qui si apre una chiara bipartizione tra chi si disorienta e non sa cosa fare del tempo ritrovato, riconoscendolo superfluo e noioso, e chi invece ne apprezza l'utilità: «Ho riscoperto quante cose si possano fare in una sola mattinata durante la quale, di solito, non faccio in tempo a fare nulla perché, tra una cosa e l'altra, sto sempre ad aprire i social e applicazioni varie» (Te4219). Certo che «non essere più abituati ad annoiarsi» e la fatica di «dover utilizzare il tempo per me», testimoniano di quanto effettivamente i social popolino le giornate di diversivi, anche se, come sostiene qualcuno, si tratta di cose inutili o superflue:

Sono davvero delusa. Non so bene cosa mi aspettassi dal mio ritorno sul web ma non c'è nulla di interessante, prima della mia settimana di astinenza, nei momenti di noia mi sembrava tutto così interessante, solo adesso mi rendo conto delle stupidaggini inutili che guardavo. Ho acceso i social da 10 minuti e già non li voglio più guardare (Sa52-19)

L'ansia e la tensione che, per alcuni, accompagnano la perdita di contatto con i media, per questa ragazza sono invece fini a loro stesse poiché si rende conto, alla prova dei fatti, che il tempo perduto non è quello che residua dall'astinenza, ma era quello dedicato ai social e alle app prima. Sono però casi minoritari, un buon numero di studenti e studentesse lamenta la fatica di sopportare un'impresa che produce sintomi fastidiosi e inediti.

[l'astinenza] ha creato in me una vera e propria dipendenza e ha determinato uno stato di instabilità mentale mai provato prima d'ora (Te43-21).

(...) mi sento triste, solo, abbandonato. Prendo in mano il cellulare, lo sblocco, comincio a guardarlo, guardo le varie icone colorate, vorrei aprirle per stare meglio. (...), come se guardando le migliaia di messaggi arrivati, da migliaia di gruppi, potessi sentirmi meno avvilito e abbattuto! (Te8-19).

Non ce la faccio più, mi sento senza qualcosa di essenziale che di solito mi completava soddisfacentemente la giornata (Sa48-19).

Senza i social soprattutto mi sembra di vivere in un vortice di solitudine (Sa78-19).

L’ambivalenza si ripresenta qui con la solita doppia funzione, ciò che per taluni avvia processi di smarrimento, depressione e di vera e propria sofferenza, in altri produce consapevolezza della vacuità degli usi consolidati e a volte ossessivi dei media che riempiono il tempo in modo futile. La loro 'mancanza' è una via di emanci- 
pazione, almeno mentale, oppure un calvario, e a ciascuna delle due soluzioni ci si avvicina scoprendole senza aver avuto prima delle chiare avvisaglie. Sentirsi sempre più soli o avviare conversazioni casuali con sconosciuti; affrontare le giornate più riposati e sereni o contare le ore che separano dalla fine dell'esperimento; stare fisicamente male immersi nei propri pensieri o godersi un insieme impensabile di novità; studiare in modo più concentrato o esserne incapaci senza le pause con notifiche e YouTube, definisce non soltanto un diverso rapporto consolidato con i media, ma strutture caratteriali, ambienti sociali e familiari differenti che orientano verso l'una o l'altra percezione.

\subsection{L'esperienza.}

«È un'esperienza che ripeterei? No. È un'esperienza che consiglierei di fare? Sì (Sa27-19).

Soluzione salomonica alla domanda conclusiva che molti si pongono nelle ultime righe dell'elaborato. Sono rarissime e quasi inesistenti le risposte entusiaste, poche quelle possibiliste «è stata comunque un'esperienza interessante che, se tornassi indietro, rifarei» (Sa58-18), mentre quella mediana, espressa in forme diverse, è più comune. Soddisfa, infatti, sia la sensazione di aver compiuto un piccolo viaggio all'inferno che a qualcosa è servito, sia $\mathrm{i}$ buoni propositi che dovrebbero scaturirne e che si sa di non poter rispettare. Quindi la scissione tra un me consapevole, ma stanco, che non ha intenzione di ripetere la prova, e un tu ancora ignaro e bisognoso di illuminazione, che invece sarebbe bene la intraprendesse, sembra la soluzione retorica perfetta per trarsi d'impaccio.

All'opposto fioccano le conclusioni felici di una «tortura psicologica (Sa36-20)», che «non rifarei» (Te4321), infatti «non vedo l'ora che arrivi la mezzanotte di stasera, perché la cosa si sta facendo pesante» (Sa4419). Si contano le ore e ci si libera usando il maiuscolo trionfale che grida: «HO ACCESO FINALMENTE IL MIO TELEFONO, MA SOPRATTUTTO HO ACCESO INTERNET!!!!» (Sa95-19).

La sfida, insomma, per alcuni è rimasta fine a se stessa: misurarsi e vedere se si è capaci di giungere alla conclusione che ci si era prefissata. Un gioco o una gara di resistenza, il cui esito è un veloce ritorno al punto di partenza

(...) non appena terminata la settimana di astinenza, mi sono trovato di nuovo immerso nella tecnologia e ho passato la successiva settimana praticamente incollato a qualunque cosa con all'interno dei microchip (Te8-19)

La prima cosa che ho fatto venerdì mattina è stata quella di 'mettermi in pari' con gli episodi di Netflix che non ho visto e di aprire i social, comunque con un minimo di consapevolezza in più (Sa17-22)

Anche se in alcuni fa capolino una definitiva consapevolezza

Solo 522 messaggi su WhatsApp, 162 nuove mail, 47 notifiche Facebook, 20 notifiche Instagram. Facendo una media ricevo al giorno circa 107 messaggi di qualsiasi tipo e dunque vengo distratta ben 107 volte al giorno (...) Quasi quasi preferivo la settimana che ho appena passato (con alcune agevolazioni) (Sa48-19)

Ho addirittura voglia di cominciare un altro periodo di astinenza. Nessuno si è preoccupato veramente, la vita è andata avanti lo stesso, i messaggi sono tanti ma sono tutti inutili. Non mi viene nemmeno voglia di leggerli tutti, perderei solo tempo. (Sa18-20)

\section{CONCLUSIONI}

Una prima osservazione riguarda la gerarchia tra $\mathrm{i}$ media. La radio è ancora molto presente tra gli studenti, così come la televisione, anche se consumate in forme eterodosse, che prescindono dall'uso dell'elettrodomestico tradizionale. Serial di successo, show e programmi musicali passano attraverso l'immancabile smartphone, ma la presa dei format tradizionali appare ancora abbastanza intatta come riempitivo dei tempi morti e delle pause di rilassamento tra un impegno e l'altro. Il che rimette in campo le osservazioni di Dardenne (1994) sul ruolo dei vecchi media, prima della nascita del web. Sono loro a fare da substrato a una serie di effetti che, come dicevamo, si producevano anche prima più $o$ meno come li abbiamo rilevati ora. Ne consegue che il mondo dei media digitali ne esce ridimensionato, almeno dal punto di vista della sua funzione di contenitore radiotelevisivo.

Nel merito dell'esperimento, l'ambivalenza, che ponevamo come criterio principale per interpretarne gli esiti, si conferma il solo approccio con cui poterne cogliere la complessità. Vi sono, e lo abbiamo visto, delle tendenze comportamentali, emotive, psicologiche che emergono chiaramente. Tuttavia, queste si presentano in relazioni duali, che comprendono degli opposti sovente tra loro inconciliabili. Se vi sono segni di una sorta di 'dipendenza' che produce ansia, depressione, 'vuoto' in alcuni, ce ne sono altri che testimoniano maggior concentrazione, libertà di movimento ed esplorazione delle proprie capacità di agency. Ne ricaviamo la conclusione che l'ambivalenza tenda a manifestarsi per soggetto, piuttosto che a convivere nella stessa persona, specializzandosi individualmente. L'attaccamento ai media vecchi e nuovi o la dipendenza che connota l'interpretazione che 
alcuni studenti vi attribuiscono, varia in ragione dell'esperienza acquisita nel loro uso e in rapporto al tipo di bisogni che con essi vengono soddisfatti. Impegnare il tempo vuoto piuttosto che riorganizzarlo; sentirsi più concentrati nello studio anziché distratti; soffrire l'ansia e il disorientamento della privazione, invece di cogliere un'opportunità; queste e altre polarizzazioni tratteggiano l'ambito di una presa di coscienza che produce forme diverse di consapevolezza. Tanto che non sarebbe male proporre esperimenti di astinenza, nelle dovute maniere e con i necessari adeguamenti, come un'attività compresa nel curriculum scolastico ${ }^{7}$ allo scopo di offrire agli studenti e alle studentesse l'opportunità di misurarsi con i rischi e le responsabilità che il web impone.

\section{RIFERIMENTI BIBLIOGRAFICI}

Ahn, J., Jung, Y. (2016). The common sense of dependence on smartphone: A comparison between digital natives and digital immigrants. New Media and Society, 18(7), 1236-1256. doi. org/10.1177/1461444814554902.

Baker, D. A., \& Algorta, G. P. (2016). The relationship between online social networking and depression: A systematic review of quantitative studies. Cyberpsychology, Behavior, and Social Networking, 19(11), 638648. doi.org/10.1089/cyber.2016.0206

Ball-Rokeach, S. J., \& DeFleur, M. L. (1976). A dependency model of mass-media effects. Communication research, 3(1), 3-21. doi.org/10.1177/009365027600300101

Berelson, B., (1949). What Missing the Newspaper Means. In Lazarsfeld P. and Stanton F. (Eds), Communications Research, 1948-1949. (111-129). Harper.

Blumler, J., Katz, E. (Eds.) (1974). The Uses of Mass Communications. Current Perspectives on Gratifications Research. Sage.

boyd d., 2014. It's Complicated. Yale University Press.

Cheever, N. A., Rosen, L. D., Carrier, L. M., \& Chavez, A. (2014). Out of sight is not out of mind: The impact of restricting wireless mobile device use on anxiety levels among low, moderate and high users. Computers in Human Behavior. 37, 290-297. doi.org/10.1016/j. chb.2014.05.002

\footnotetext{
${ }^{7}$ Moeller, Powers e Roberts (2012) giustificano il loro esperimento di astinenza dai media per $24 \mathrm{~h}$, chiesto a quasi 1000 studenti di 12 università sparse nel mondo, rifacendosi al Modulo 7, Unità 1 del Curriculum UNESCO relativo alle "Internet Opportunities and Challenges», dove, tra l'altro, si sottolinea la necessità che i docenti si impegnino a «Develop their ability to use educational methods and basic tools to help young people use the Internet responsibly - and make them aware of the related opportunities, challenges and risks». http://unesco.mil-forteachers.unaoc.org/modules/module-7/unit-1/
}

Clayton, R. B., Leshner, G., \& Almond, A. (2015). The extended iSelf: The impact of iPhone separation on cognition, emotion, and physiology. Journal of Computer-Mediated Communication, 20(2), 119-135. doi. org/10.1111/jcc4.12109

Chou, C., Condron, L., \& Belland, J. C. (2005). A review of the research on Internet addiction. Educational psychology review, 17(4), 363-388. doi.org/10.1007/ s10648-005-8138-1

Cohen, A. A. (1981). People without media: Attitudes and behavior during a general media strike. Journal of Broadcasting \& Electronic Media, 25(2), 171-180. doi.org/10.1080/08838158109386440

Dardenne, R. (1994). Student Musings on Life without Mass Media: Antidote for Silence, Boredom, and Thinking. The Journalism Educator, 49(3), 72-79. doi. org/10.1177/107769589404900310

De Bock, H. (1980). Gratification frustration during a newspaper strike and a TV blackout. Journalism Quarterly, 57(1), 61-78. doi.org/10.1177/107769908005700109

Elliott, W. R., \& Rosenberg, W. L. (1987). The 1985 Philadelphia newspaper strike: A uses and gratifications study. Journalism Quarterly, 64, 679-687. doi. org/10.1177/107769908706400401

Grossi, G. (2017). Cultura e ambivalenza. Il campo culturale nel XXI secolo: dilemmi e ipotesi. Quaderni di Sociologia, 73, 81-105. doi.org/10.4000/qds.1664

Hall, J. A., Xing, C., Ross, E. M., \& Johnson, R. M. (2021). Experimentally manipulating social media abstinence: results of a four-week diary study. Media Psychology, 24(2), 259-275. doi.org/10.1080/15213269 .2019 .1688171

Hoffner, C. A., Lee, S., \& Park, S. J. (2016). "I miss my mobile phone!": Self-expansion via mobile phone and responses to phone loss. New Media \& Society, 18(11), 2452-2468. doi.org/10.1177/1461444815592665

Knowles, A. D., Fenner, P., McNeil, M., Merrigan, C., \& Power, V. (1989). TV or not TV: the impact of two weeks without television. Media Information Australia, 52(1), 26-29. doi.org/10.1177/1329878X8905200107

Lee, U., Yang, S., Ko, M., \& Lee, J. (2014). Supporting temporary non-use of smartphones. In ACM SIGCHI Conference on Human Factors in Computing Systems (CHI14). Workshop: Refusing, Limitng, Departing.

Lepp, A., Barkley, J. E., \& Karpinski, A. C. (2014). The relationship between cell phone use, academic performance, anxiety, and satisfaction with life in college students. Computers in Human Behavior, 31, 343-350. doi.org/10.1016/j.chb.2013.10.049

Mascheroni, G., Vincent, J. (2016). Perpetual contact as a communicative affordance: Opportunities, constraints, 
and emotions. Mobile Media and Communication. 4, 3, 310-326. doi.org/10.1177/2050157916639347

Moeller, S., Powers, E., \& Roberts, J. (2012). «The world unplugged» and «24 hours without media»: Media literacy to develop self-awareness regarding media. Comunicar. Media Education Research Journal, 20(2). DOI: 10.3916/C39-2012-02-04

Paasonen, S. (2021). Dependent, Distracted, Bored: Affective Formations in Networked Media. MIT Press.

Rubin, A. M., \& Windahl, S. (1986). The uses and dependency model of mass communication. Critical Studies in Media Communication, 3(2), 184-199. doi. org/10.1080/15295039609366643

Scarcelli, C. M. (2019). Lo smartphone oltre la dipendenza, in Drusian, M., Magaudda, P., \& Scarcelli, M. (Eds). Vite interconnesse. Pratiche digitali attraverso app, smartphone e piattaforme online. Meltemi.

Silva, S. (2012) On emotion and memories: the consumption of mobile phones as "affective technology." International Review of Social Research, 2, 157-172. DOI: 10.1515/irsr-2012-0011

Stieger, S., \& Lewetz, D. (2018). A week without using social media: Results from an ecological momentary intervention study using smartphones. Cyberpsychology, Behavior, and Social Networking, 21(10), 618624. doi.org/10.1089/cyber.2018.0070

Tan, A. S. (1977). Why TV is missed: A functional analysis. Journal of Broadcasting \& Electronic Media, 21(3), 371-380. doi.org/10.1080/08838157709363845

Tromholt, M. (2016). The Facebook experiment: Quitting Facebook leads to higher levels of well-being. Cyberpsychology, behavior, and social networking, 19(11), 661-666. doi.org/10.1089/cyber.2016.0259

Vincent, J. (2006). Emotional attachment and mobile phones. Knowledge, Technology \& Policy, 19, 39-44. doi.org/10.1007/s12130-006-1013-7

Windahl, S., Höjerback, I., \& Hedinsson, E. (1986). Adolescents without television: A study in media deprivation. Journal of Broadcasting \& Electronic Media, 30(1), 47-63. doi.org/10.1080/08838158609386607 